\section{Ideación suicida en personal de salud durante la pandemia del COVID-19; un problema desatendido en Bolivia. $d$}

iD Yercin Mamani Ortiz

\section{Señor Editor:}

Mediante la presente, tengo a bien compartir una breve contextualización sobre los resultados obtenidos por el equipo del estudio "The COVID-19 HEalth caRe wOrkErs Study (HÉROES)" (1) en colaboración entre la Organización Panamericana de la Salud (OPS), durante el año 2020 con el objetivo de analizar el estado de la salud mental de los equipos de salud que han hecho su trabajo durante la pandemia de COVID-19 en la Región de las Américas.

Los resultados de esta colaboración se resumen en un reporte publicado el mes de enero del 2022 y difundido a través de seminarios en línea, así como conferencias de prensa, sin embargo, no ha tenido un eco en las autoridades nacionales en salud, a pesar de que los resultados referidos para Bolivia son por demás preocupantes.

El reporte refleja la situación de la salud mental del personal de salud de 11 países de la región de Latino América y el Caribe (LAC), mediante la aplicación de cuestionarios autoaplicados estandarizados y de validez ampliamente estudiados, considerando como variables de resultado: 1) Malestar psicológico, 2) Síntomas depresivos, 3) Pensamientos suicidas y 4) factores de riesgo y protectores asociados a las tres variables principales. En el caso de Bolivia participaron 167 personas de 12 establecimientos de salud, seleccionados mediante un muestreo polietápico.

Los resultados más importantes para Bolivia reflejan que 95\% de los participantes correspondían al sistema público de salud, el $51 \%$ de ellos comprendidos menores o igual a los 35 años; $33 \%$ con estudios con respecto a postgrado, $51 \%$ pregrado, $16 \%$ técnicos; resaltando que fue el único país que reportó personal con escuela primaria incompleta igual a 3\%. En relación con la salud mental de los equipos de salud, Bolivia ocupó el 2. ${ }^{\circ}$ intervalo más alto, siendo Colombia que ocupó el nivel más alto. Respecto a los síntomas depresivos, la sospecha de depresión en los participantes de Bolivia fue el más alto 29 a $36 \%$; seguido de Chile 22 a 29\%. Finalmente, y no menos relevante, la proporción de personal de salud con ideación suicida fue el más alto en Bolivia y Chile en comparación al resto de países de la región de $\operatorname{LAC}(14,7$ a 17,90\%). Entre los factores de riesgo más importantes resaltaron, la necesidad de apoyo social, el estar preocupado porque los seres queridos contraigan COVID-19,
Correspondencia a:

1 Médico Salubrista; Investigador en Instituto de Investigaciones Biomédicas e Investigación Social Medicina (IIBISMED) de la Universidad Mayor de San Simón (UMSS).

Cochabamba, Bolivia.

Email de contacto:

ye.mamani@umss.edu

Procedencia y arbitraje:

No comisionado, sometido a arbitraje externo

Recibido para publicación: 11 de febrero del 2022

Aceptado para publicación: 03 de marzo del 2022

Citar como:

Mamani Ortiz Y. Ideación suicida en personal de salud durante la pandemia del COVID-19; un problema desatendido en Bolivia. Recisa UNITEPC. 2022; 9(1):09-11.

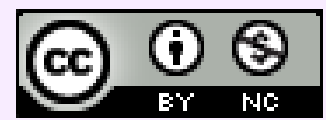

Esta obra está bajo una Licencia Creative Commons Atribución-NoComercial 4.0 Internacional. 
ISSN $2520-9825$

Ios conflictos con familiares de pacientes con COVID-19, los cambios de funciones, la estigmatización por trabajar con pacientes con COVID-19 y haber experimentado violencia por trabajar con pacientes con COVID-19.

Estos hallazgos deben llamar a la reflexión a las autoridades en salud de nuestro país, así como a las autoridades políticas a nivel municipal, departamental y nacional; debido a que se refleja de manera alarmante que al menos 1 de cada 10 trabajadores en salud presentaron pensamientos de ideación suicida durante la pandemia del COVID-19 en nuestro país. Estos resultados son un reflejo de la actual situación del sistema sanitario en Bolivia, que fue colapsada durante la primera, $3 .^{a}$ y $4 .^{a}$ ola de la pandemia por COVID-19. Vanos fueron los esfuerzos de ampliar la capacidad de respuesta hospitalaria, promovidos en el caso de Cochabamba desde la "Sala Situacional" (2), en el que se promovieron estrategias que incluían la ampliación física de los ambientes, tanto en el sistema público como en la seguridad social; la reconversión de servicios, la implementación de estrategias de vigilancia comunitaria activa, entre otras; pero que lamentablemente no se acompañaban de un incremento en el número de personal de salud de manera permanente. A mediados de la primera ola, se inició con la contratación de personal temporal por el lapso de 3 meses, los mismos que fueron recontratados periódicamente en cada ola; sin embargo, hasta la fecha estos no pudieron ser consolidados como ítems de nueva creación, a pesar de que en diciembre del 2020 se aprobara la ley nacional que establece un presupuesto del $10 \%$ del Producto Interno Bruto (PIB) para salud, con el objetivo de incrementar el número de ítems en todos los departamentos, mismo que fue comprometido por todos los candidatos a las elecciones nacionales y departamentales, pero que a la fecha no tuvieron ningún resultado favorable.

Son varios los factores contextuales que pudiesen haber incidido en estas prevalencias tan altas en comparación a otros países de la región de LAC; siendo necesarios la generación de investigaciones locales que permitan una mejor comprensión del comportamiento clínico y epidemiológico de la salud mental del personal de salud en el contexto boliviano; contemplando los diferentes aspectos críticos, entre los que se pueden destacar:

- La brecha de recursos humanos y su relación con la saturación del sistema sanitario.

- Los cambios organizacionales para la atención de pacientes con COVID-19 y su impacto en la productividad de los servicios.

- La inestabilidad laboral del personal de salud y su asociación con la calidad de vida laboral.

- EL proceso de vacunación y su impacto en la morbilidad y mortalidad por COVID-19 en personal de salud.

- La capacidad de resiliencia del sistema de salud frente a situaciones de estrés institucional en situaciones de epidemia.

Para nadie es desconocido que la pandemia por COVID-19 ha generado un aumento en los requerimientos de atención por parte de la población, provocando una sobrecarga asistencial en los sistemas de salud, agravada por las bajas temporales 0 definitivas generadas por la morbilidad y mortalidad por COVID-19; por lo que se hace imperativo la protección de la salud mental, mejorar sus condiciones contractuales de trabajo, una remuneración digna, así como el apoyo social en el lugar de trabajo. 
Los resultados del estudio internacional HÉROES muestran un índice alto de ideación suicida en Bolivia, en comparación otros países de la región. Por ello, se requiere documentar el impacto de la pandemia del COVID-19 en el personal de salud, de manera que sirvan de sustento técnico en la generación de estrategias, planes de contención y políticas de salud que permitan minimizar los efectos a largo plazo; considerando que las consecuencias de la pandemia de COVID-19 sobre la salud mental del personal sanitario sean sostenidas en el tiempo.

\section{Referencias bibliográficas}

1. OPS, Organización Panamericana de la salud, The COVID-19 HEalth caRe wOrkErs Study (HÉROES). Informe Regional de las Américas, OPS/NMH/MH/ COVID-19/22-0001, 2022 Accedido él: 04-02-2022. Disponible en: https://iris.paho. org/handle/10665.2/55563.

2. Mamani Ortiz Y, Siles Toledo AH. La Sala Situacional "Cochabamba Somos Todos", una respuesta técnica en la lucha contra el COVID-19. Gac Med Bol " 2021; 44. (1): 128-130. 